\title{
Knowledge extraction to automate CFD analysis in abdominal aneurysm diagnosis and treatment
}

\author{
Simone Bartesaghi* and Giorgio Colombo \\ Dipartimento di Meccanica, \\ Politecnico di Milano, \\ Via G. La Masa, 1, 20156 Milano, Italy \\ E-mail: simone.bartesaghi@mail.polimi.it \\ E-mail: giorgio.colombo@mecc.polimi.it \\ *Corresponding author
}

\begin{abstract}
In the last few years, advanced simulation tools have been tested in academic researches to evaluate the causes and evolution of abdominal aortic aneurysm (AAA). This study describes the activity of knowledge extraction aimed at automating the CFD analysis of an AAA. A simple benchmark geometry is considered. Knowledge and rules extraction are done by comparing: 2D and 3D CFD solutions, results from Newtonian and non-Newtonian formulations, influence of the prism layer and inlet boundary conditions, and numerical solver schemes. A grid refinement study using the verification and validation approach was done. As a result, finer hexahedral meshes with a prism layer near the vessel walls is necessary to capture the velocity gradient and wall shear stress correctly; a simple Newtonian formulation is enough to capture the fluid flow behaviour. A first step in the validation process is taken through the application of the extracted rules on a real patient's specific geometries.
\end{abstract}

Keywords: haemodynamic; aneurysm; diagnosis; therapy; computational fluid dynamics; CFD.

Biographical notes: Simone Bartesaghi holds the current position of $\mathrm{PhD}$ candidate at the Politecnico di Milano, Dipartimento di Meccanica. $\mathrm{He}$ is responsible for $\mathrm{KB} / \mathrm{Embedded} \mathrm{CFD}$ simulations in the biomedical filed. He is also a member of Yacht and Superyacht Research Group of the School of Marine Science and Technology of the Newcastle University. His previous experience includes the specialist degree in Mechanical Engineering at Politecnico di Milano, with the qualification of Mechanical Engineer specialised in methods and techniques of virtual prototyping. He attended a Master in Yacht Design VIII ed. (110/110) at the Politecnico di Milano and Università degli Studi di Genova.

Giorgio Colombo is an Associate Professor at the Politecnico di Milano. In 1989, he started his research activities at ITIA-CNR in Milan. From 1992 to 2000, he has been an Assistant Professor at the Faculty of Engineering of Parma. Since 2001, he has been an Associate Professor. His research activities focus on: shape modelling, knowledge-based engineering, computer aided design, simulation techniques, computer graphics, virtual reality, and robotics. He has been a coordinator and responsible for the research unit activities in national and European research projects. 


\section{Introduction}

Up to today simulations have been playing an important role in industrial processes. However, they have had a limited impact - or not at all - on many other problems, which may benefit from these techniques. Especially, they may facilitate decision making, in many fields. Scientific literature cases are reported, concerning the estimation of air quality with pollution sources near building or industrial sites (Huber et al., 2004) in order to prevent damages caused by wind borne debris (Kakimpa et al., 2009) or to reduce emission combustion for aero-engines (Pegemanyfar et al., 2008). From this point of view, a very important field where simulations can help decision making is health.

Various types of diseases and stressful lifestyle increase human body vulnerability; abdominal aortic aneurysm (AAA) is a serious one, since it can be fatal if it breaks. It is related to the blood circulatory system at the level of abdomen and affects $5 \%$ of men and $1 \%$ of women over the age of 65 . In particular, an AAA weakens the walls of the blood vessel; such an alteration of blood vessels may lead to bad consequences whenever it causes the rupture of the vessel itself and then a serious haemorrhage (Karmonik et al., 2008). In most cases, $80 \%$ to $90 \%$ (prehospital deaths included), the rupture is fatal, while elective repair should be considered only for any AAA with a maximal diameter of $5.5 \mathrm{~cm}$ (men) or $5.0 \mathrm{~cm}$ (women).

The only possible treatment of AAA is surgery; the evaluation of a possible surgery is performed by physicians after carrying out analysis on biomedical images, obtained from scanning systems of the human body. But not always surgery is urgent or necessary, while in other cases the problem may be underestimated. It follows that decision making can be easier and more accurate if some risk indicators or risk scores are available. Over the past years several risk rupture indicators have been developed, like Anatomical Indicators (maximum diameter of AAA) or Structural Indicators (PWS, PWRR, GWSS). However, today there are no medical device systems able to measure or evaluate the risk. Thanks to imaging techniques and computational geometry, today it is possible to perform simulations on realistic geometries and on real blood flow conditions. Computational fluid dynamics (CFD) tools can be used to compute haemodynamic indicators [aneurysm formation index (AFI), gradient oscillating number (GON), relative residence time (RRT), oscillatory shear index (OSI), etc.] directly from the medical images.

In this sense, 3D CFD techniques are decisively improved and they permit studies related to local haemodynamics in the biomechanics processes of the vascular bed and allow the testing and validation of both clinical and surgical procedures. But the evolution of computing technologies has not yet been used in clinical practice and CFD simulations are up to now relegated in university institutes or research centres. Main causes may be: 
- Lack of CFD simulations experts in hospital laboratories and the difficulty to find structures which provide a service of this type.

- CFD simulations require significant resources, in terms of human experts, hardware, software, and financial. They are technically complex and require high computation times;

- Medical professionals' scepticism: they do not probably consider simulation techniques adequate to support the diagnosis and treatment of such a disease.

These limits can be exceeded by integrating in an automatic mode CFD simulations on the process to obtain medical images. In such an approach, we can consider a direct process from medical imaging to risk indicator evaluation. To do that, it is necessary to automate all the activities from image acquisition to CAD geometry reconstruction, CFD pre-processing, computing, post-processing and reporting.

CFD and traditional tools are then integrated. As a result, it has been possible to perform CFD analysis based on medical imaging and flow data. Compared to other diagnostic tools, CFD methods show more advantages. First of all, they are less expensive, give a detailed representation of the velocity vectors and pressure fields, allow easier parametric studies based on geometric or flow quantities, and above all they are more ethical since they are not performed on patients.

\subsection{CFD in blood flow simulation}

CFD techniques have been investigated in academic researches as an aid to the investigation and the prognosis of such a pathology - see as an example in Boutsianis et al. (2009). The use of computational tools applied to blood flow in human arteries is increasing. Nowadays, these tools provide detailed and trustable information on haemodynamic properties so that researchers can investigate a large number of problems which have been hardly treatable with traditional engineering flow measurement techniques. On the one hand, during the ' $80 \mathrm{~s}$, a powerful innovative tool to study haemodynamics quantities and indices appeared: the CFD. On the other hand, other tools - such as magnetic resonance imaging (MRI), Doppler ultrasound and X-ray cine-angiography, keep on being used and allow the investigation in vivo of the blood flow dynamics as well as the highlighting of the differences among haemodynamics quantities of different people.

Recent works tackle the problem through CFD methods, and the article mentioned in Boutsianis et al. (2009) is an example; other examples reported by Botar et al. (2010) and Ford et al. (2008) show the accuracy of the CFD, performing a validation of the method by using experimental data and biomedical analysis. Using in vivo experimental data, Karmonik et al. (2008) reported a good quality of results from CFD with patient-specific boundary conditions as input to numerical simulation. Three-phase simulation of blood flow was performed by Jung and Hassanein (2008); they showed the capability of CFD to provide information about the interaction between red blood cells and leukocytes. See also Yilmaz and Gundogdu (2009) for specific application of CFD as a tool of haemodynamic simulation.

To study the stress related to vessel walls, CFD is coupled with FEM through fluid structure interaction (FSI), see Valencia et al. (2008). These examples and many others (for example, Petkova et al., 2003) show that up to today the approach has been 
developed in a vertical way, giving precedence to the refinement of models and techniques for the specific problems; while the horizontal development, linked to the integration between models and tools in order to support decision making, has been neglected.

\subsection{Embedded CFD approach}

Approaches and strategies of integration between $\mathrm{CAD}, \mathrm{CAE}$ and product development were presented and discussed in different contexts. These analysed works successfully show the process of integration between various technologies, from the haemodynamic flow simulation to the design in aerospace field. What emerges is not a clear definition of basic rules that allow the process to operate in embedded mode, without the intervention of a specialist in the development and workflow analysis of the product. These rules are mainly to be found in the process to develop the CAD model ready for a CFD or FEM model. It is necessary to explain these rules and why we use them.

Examples of 'embedded simulations' emerge in other contexts, for example in the design automation domain, above all in aeronautic and automotive fields. Indeed there are applications in which structural components are automatically dimensioned and verified through finite elements analysis. The workflow to develop a blade in embedded mode is explained in Andersson et al. (2006) where a rotor blade is designed, simulated and optimised by using CAD models and FEM tools. The knowledge in FEM is widely used to develop automotive components (Chapman and Pinfold, 2001) or in aeronautic industry to design and optimise airplane wings (Cooper et al., 2009). Other examples of knowledge-based approaches and embedded simulations are provided in Mohammed et al. (2008) and Nawijn et al. (2006).

An example of knowledge-based procedure in blood simulation is found in Deschamps et al. (2004), where an embedded boundary method is applied to reconstruct a geometry from medical images.

In this work, the field of interest is the automatic process as well as the virtualisation and knowledge representation of the entire workflow from medical imaging to 3D visualisation of haemodynamics risk indicators. The relevance of the research is to define a strategy to automate the process in order to reach an integration between traditional clinical diagnostics tools (imaging) and modern computer aided engineering (CAE)-based tools, in this case CFD tools. The latter is the innovative feature of the research. As far as the methodological approach is concerned, state of the art tools and methods have been validated and integrated to work in an embedded process.

The automatic workflow for risk evaluation in the AAA disease is entirely based on the domain knowledge representation. Figure 1 shows the workflow proposed in this work; every task is performed by using automatic/optimal procedures. Embedded algorithms are designed to create a bridge between every part of the workflow. In particular, embedded algorithms work based on rules, information and procedures which are extracted from a detailed systematic analysis of each phase of the workflow; methods and protocols have been designed and developed in order to reach the accuracy required.

The study is mainly focused on the grid topology, discretization rules and spatial dimension of the problem (2D or 3D) useful to define an automatic pre-processing strategy for these applications. The assessment of the pressure field, the velocity in the vessel and the consequent stresses on the vessel fabric are important parameters to know 
in order to facilitate the prognosis, the risk and the choice of the therapy. There are many reports in the scientific literature regarding such cases.

Concerning the LifeScience, this work will face some fundamental points, useful to plan the problem of implementing a prototype based on 'Embedded CFD' (Milašinović et al., 2008). It is useful to assess the risk and to decide therapies in such cases. It is worth underlining that we leave out the issue concerning the integration between medical tools and CFD simulation. The main innovation and contribution of this research project is related to the extraction of procedures, rules and knowledge to automate the pre-processing phase for a robust automatic mesh generation. Rules for meshing are based on the geometric properties of the aorta vessel. The mesh parameters extracted by the investigation are normalised to the aorta diameter and are at the base of the mesh generation process. All the other parameters in the pre-processing phase are driven by the mesh base size.

\section{Approach, method and tools for knowledge extraction}

\subsection{Approach}

An approach based on 'embedded CFD analysis' has to follow some steps:

1 automatic reconstruction of the geometric model of the vessel's trait hit by the aneurysm, based on the medical images taken from diagnosis system

2 pre-processing phase managed in automatic mode through the application of rules

3 solving phase done by one or more solvers (in the case of CFD or FSI)

4 post-processing phase, automatically managed through a rule system to produce ready-to-use information.

Figure 1(a) through IDEF diagram representation, shows the workflow of a standard medical diagnosis. Figure 1(b) adds, in support of medical diagnosis, the CFD simulation. CFD simulation, Figure 1(c), must be integrated in the process of diagnosis and must be able to ensure a diagnostic support without the intervention of a CFD specialist. Every step of the numerical simulation must be based on specific knowledge.

For an automatic CFD analysis, the required knowledge is related in all parts of the process. The pre-processing needs to be fully automatic and intelligent. It is necessary to know the geometric dimensions of the aorta vessel and relate the mesh dimension to these properties. The influence of the boundary condition is a key point for a CFD user: inlet and outlets must be placed to ensure a complete development of the blood flow in the aneurysm zone. Because of the automatic process, the prism layer generation is automatic and the knowledge is required to know if this feature is significantly influencing the solution. Part of the prism layer knowledge is the minimum number of prisms in the near wall layer. Regarding the solver, knowledge is necessary to define the time step size and the internal iteration of the solver per time step. Moreover, the definition of the fluid rheology is subjected to knowledge. The reporting phase must be also automatic and knowledge-based: it is necessary to know which scalar quantities are important for the visualisation, integrate them over the simulation time and provide an easy-to-use representation of the haemodynamics indices to aid AAA diagnosis. 
Figure 1 IDEF of the process workflow, (a) conventional workflow in medical analysis (b) CFD-aided medical analysis workflow (c) CFD embedded workflow

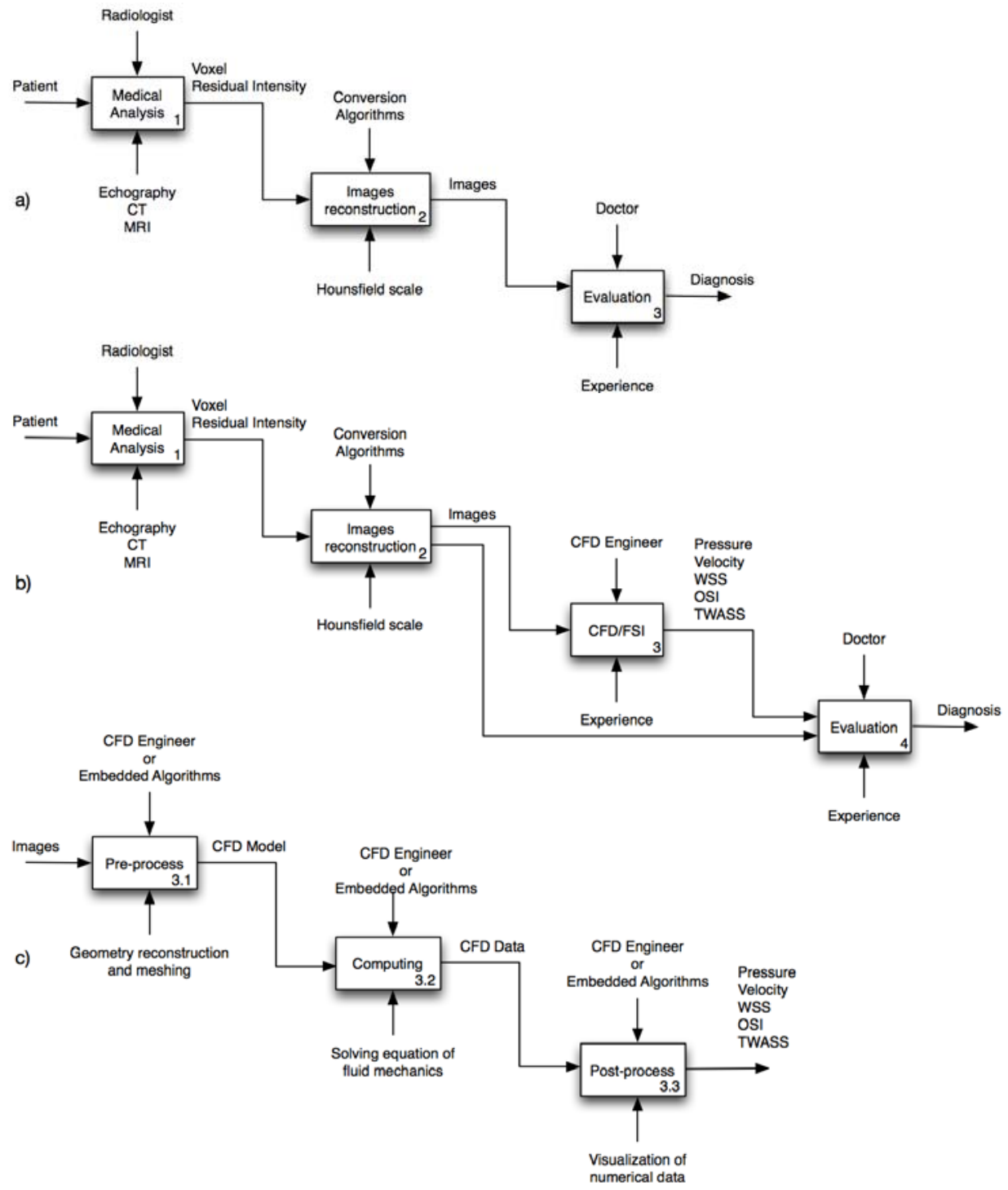

\subsection{Method}

The main goal of this research project is to extract rules and information useful to automate simulation of blood flow inside vessels afflicted by an aneurysm. First of all, in CFD simulations, some points must be stated, concerning: 
- $\quad$ geometry and CAD model

- finite volume representation

- $\quad$ equation to be solved.

Problems related to geometry and CAD model are not discussed in this work. The first investigation is focused on the choice of the spatial dimension of the problem; the vessel geometry is a 3D CAD model and the first task is to understand the possibility to reduce the order of complexity of the problem to a $2 \mathrm{D}$ equivalent geometry. A comparative study between 3D and 1D models in blood flow simulation was performed by Grindberg et al. (2011). To reduce the computational effort, in appropriate conditions it is possible to decrease the order of the spatial dimension of the model. Some works in industrial applications face up the problems related to the simplification of the model dimension (Arghiropol and Rotaru, 2010; Moroz et al., 2005). A 3D geometry and its related tridimensional calculation grid takes longer calculation times than a 2D grid. Obviously, the results are different; the problem is the quantification of such gap and to find out a strategy which allows adopting reduced geometric models and computational grids. Others have investigated the problem of the discretization (Longest and Vinchurkar, 2006; Spiegel et al., 2011) and the effect of the geometrical variations, vessel geometry-sensitive (diameter, etc.) (Celi et al., 2009).

The method used for knowledge extraction is based on a systematic investigation of each parameter subjected to the knowledge. For the pre-processing phase, using a parametric benchmark model, knowledge is extracted regarding the influence of the geometry and the correlation between the two spatial dimensions, 2D vs. 3D simulations (4.1). By using the verification and validation procedure, with the Richardson extrapolation and Grid Convergence Index (GCI), topology and geometry related mesh are achieved and knowledge about normalised mesh size is extracted for the setup of the automatic pre-processing phase (4.2). Also, the influence of the fluid viscosity is taken into account; a simple Newtonian formulation is compared to a power-law non-Newtonian formulation for the blood (4.3) to verify the weight of the mathematical formulation on the overall solution. A systematic investigation on the number of prisms in the near wall region allows the extraction of knowledge of the treatment of this zone comparing the wall shear stress (WSS) magnitude convergence (4.4).

The influence of the boundary conditions is evaluated in terms of WSS and fully developed flow in the aneurysm. Using the pipe flow theory, a normalised development length is extracted to use for AAA problems. Knowledge is finally extracted by comparing solutions with different position of the inlet-outlets boundary conditions, with and without boundary extension to ensure a complete development of the fluid flow into the aneurysmatic zone. The aim is to understand how great is the influence of the boundary condition on the global scalar quantities useful for diagnosis and treatment (4.5).

All the knowledge extracted by the previous task can be used to setup a (semi)automatic workflow for the pre-processing phase to reduce human resources and time effort for CFD simulations of AAA

The final investigation is to compare numerical results from CFD simulations of a real cardiac cycle using the same setup but different solvers, one commercial and one open source, to extract a basic knowledge about the choice of the solver. 


\subsection{Tools and materials}

For the study of problems related to CFD simulation, a reference geometry was employed to highlight the object of interest (Arslan et al., 2005). This model allows us to simplify the problem for the study of automatic analysis. Figure 2 shows the 3D CAD model of an artery with an aneurysm located on the section LCA. The CAD model is able to represent the main stretch coronary (LAC), bifurcations (LAD, LCX) and the presence of an aneurysm. The area affected by aneurysm is shaped like a sphere, while the arteries are modelled as circular ducts with constant section, with no change of curvature. The CAD model thus configured can be considered as a benchmark. The diameter of the traits LAC, LAD, LCX is $6 \mathrm{~mm}$, while the diameter of the aneurysm is $20 \mathrm{~mm}$. LAD and LCX traits form between them an angle of $60^{\circ}$. Wessel walls were assumed to be rigid and impermeable. The $2 \mathrm{D}$ model was derived from the $3 \mathrm{D}$ model considering only the average-cutting plane of the three-dimensional geometry. For the comparison of $3 \mathrm{D}$ and $2 \mathrm{D}$ simulation, the CAD geometry was reconstructed as parametric model. In embedded mode was possible to change the geometric dimension of the LCA diameter, LCX and LAD diameter, aneurysm diameter and angle between the traits LCX/LAD. Thirty different combinations of geometrical parameters were tested for $3 \mathrm{D}$ and related 2D simulations. Grids were generated by using automatic discretization algorithms based on the knowledge of the considered geometry. All models were simulated by using hybrid grids, consisting of a prismatic layer near the vessel wall and multi-faceted elements (tetrahedral, polyhedral or hexahedral) in the remaining volume control. This allowed us to automate the pre-processing and to generate meshes with local refinements congruent with each other according to the morphological characteristics of the geometry.

The fluid dynamic equations, which describe the velocity and pressure fields are known as Navier-Stokes equations (Navier, 1823; Stokes, 1845). For complex geometries the equations must be solved numerically. The equations of fluid mechanics are discretized in finite volume, called control volume (CV) in which the velocity, pressure and other unknown variables are solved. For the rule extraction about the mesh generation, steady state CFD simulations were performed by using CD-Adapco's Star-CCM+ commercial RANS code. Coupling between pressure and velocity is doing by a segregated solver. Discretization of the equation is performed using a $2^{\text {nd }}$ order numerical scheme. No turbulence models are used and the solver setup is for laminar fluid flow. For the comparison between different solvers, the simulations are performed with unsteady formulation using Star-CCM+ and OpenFOAM platforms. The numerical simulations were performed considering the blood as a Newtonian fluid at a constant speed. The properties of the fluid are shown in Table 1. For the investigation of the spatial discretization and numerical models have been tested two flow conditions, first one is an average velocity and the second one is the diastolic velocity (Arslan et al., 2005 ); for the analysis of correlation between $2 \mathrm{D}$ and $3 \mathrm{D}$ simulations a third velocity as a variational parameter has been considered, too (Table 2). 
Figure 2 CAD model of the benchmark geometry

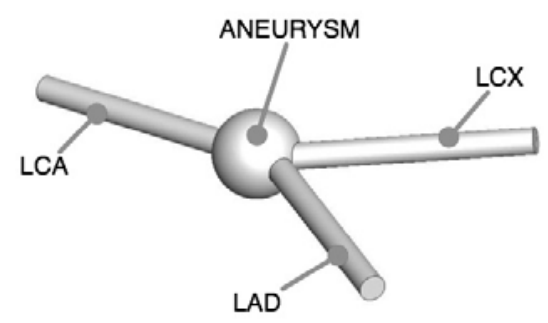

Table 1 Blood properties as Newtonian fluid

\begin{tabular}{lc}
\hline Density $\left[\mathrm{kg} / \mathrm{m}^{3}\right]$ & 1050 \\
Viscosity $[\mathrm{kg} / \mathrm{m}-\mathrm{s}]$ & 0.0035 \\
\hline
\end{tabular}

Table 2 Inlet velocity conditions tested and related Reynolds numbers

\begin{tabular}{lccc}
\hline Velocity $[\mathrm{m} / \mathrm{s}]$ & 0.1 & 0.14 & 0.25 \\
Reynolds & 180 & 250 & 450 \\
\hline
\end{tabular}

Simulations were performed on a Intel ${ }^{\circledR}$ Core $^{\mathrm{TM}}$ i7 CPU with 4.00 GB of RAM. Results of the simulations were analysed in qualitative and quantitative mode. For the qualitative mode, the contour plot of the scalar velocity magnitude inside the vessel is analysed to understand the main difference of the fluid flow. Furthermore, the velocity profile before the aneurysm and the WSS were used as quantitative parameters to perform numerical comparisons and grid verification. Figure 3 shows the boundary conditions imposed for the CFD simulations. The inlet velocity profile at the entrance was selected as a flat type and developed to the parabolic velocity profile inside the LCA. The flow distribution rates at the outlet were assumed to be $70 \%$ leaving from LAD and $30 \%$ leaving from LCX. The flow distribution rate was defined as:

$$
F R=\frac{Q_{\text {out }}}{Q_{\text {in }}}
$$

where $Q_{\text {out }}$ is the mean flow in one of the outlet coroner arteries and $Q_{\text {in }}$ is inlet mean flow rate.

Figure 3 CFD model boundary conditions (see online version for colours)

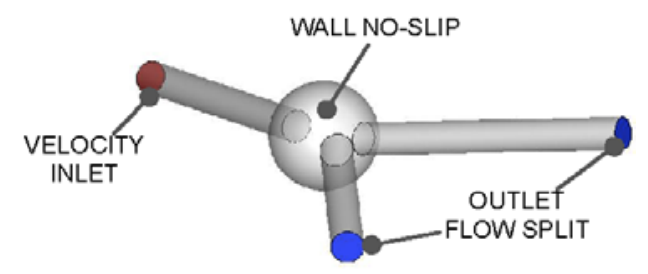




\section{Mathematical model}

In the fluid dynamics theory we analyse the solutions of the integral and differential equations. Using the fundamental laws of fluid mechanics, it is possible to derive equations able to modelling the continuous domain. Amongst all, the most important are:

- conservation of mass

- conservation of momentum

- conservation of energy.

The equations solved by using numerical methods are as follows:

$$
\left\{\begin{array}{l}
\nabla \cdot \vec{v}=0 \\
\frac{\partial \rho \vec{v}}{\partial t}+\vec{v}(\nabla \cdot \vec{v})-\rho \vec{g}+\vec{\nabla} p-\vec{\nabla} \cdot \overrightarrow{\vec{\tau}}=0
\end{array}\right.
$$

It is not necessary to define any closure model of the Navier-Stokes equations (turbulence models) because based on the physical geometrical characteristics of the problem, the flow is laminar. Moreover, the equation of conservation of energy can be neglected.

\subsection{Fluid viscosity}

In a fluid, the stress tensor $\overrightarrow{\vec{\tau}}$ depends on the efforts of the angular strain rate $\overrightarrow{\vec{S}}$. The relationship between $\overrightarrow{\vec{\tau}}$ and $\overrightarrow{\vec{S}}$ is called 'constitutive relationship of a fluid'. For those of Newtonian fluids, adopting the hypothesis of Stokes, in the case of incompressible viscous flow, with $\rho=\operatorname{cost} \mathrm{e} \mu=$ cost, this relationship is proportional and is written:

$$
\overrightarrow{\vec{\tau}}=2 \mu \overrightarrow{\vec{S}}
$$

For some fluids, called non-Newtonian, the stress tensor can be written using a term that identifies the non-Newtonian viscosity of the fluid itself $\eta$ :

$$
\overrightarrow{\vec{\tau}}=2 \eta \overrightarrow{\vec{S}}
$$

where $\eta$ is function of the three invariants of the tensor $\overrightarrow{\vec{S}}$. To model the dynamic viscosity of the non-Newtonian fluid, we used the non-Newtonian generalised power-law, where $\eta$ is a function only of the shear rate $\gamma$. The model of viscosity is defined as follows:

$$
\mu_{\min }<\left[\frac{\tau_{0}+k\left(\gamma^{n}-\tau_{0} / \mu_{0}\right)}{\gamma}\right]<\mu_{\max }
$$

where $k$ is an average value of fluid viscosity, $n$ is a coefficient (deviation of the behaviour from Newtonian type, called power-law index); $\tau_{0}$ is the yield stress threshold and $\mu_{0}$ is the yielding viscosity. When the value of $\gamma$ is less than the ratio $\tau_{0} / \mu_{0}$, the value of dynamic viscosity is equal to $\mu_{0}$, otherwise it is derived from the law of non-Newtonian fluid viscosity. 
In the carried out CFD simulations, the fluid (blood) was modelled both as a Newtonian fluid and a non-Newtonian fluid.

\subsection{Indices in hemodynamic}

There are several indices to describe the effect of haemodynamics factors on the physiological state of the vessel. These indicators may be distinguished according their location, as follows:

- wall vessel located

- $\quad$ main stream or bulk located.

As previously mentioned, sometimes the AAA diameter is not enough to make a decision. To support the decision-making process, some haemodynamics indices are employed. These indices are based on the stress status of the endothelia layer of the blood vessel. The WSS components are at the basis of these indices.

The WSS can change the morphology and orientation of the endothelial reticule the tissue that constitutes the wall of blood vessels. The haemodynamic forces play an important role in the development of diseases of the blood vessels. A mathematical definition of these indices is given through a short literature review about related values and limitations. One of the most important indices in hemodynamic is the OSI. In accordance with $\mathrm{He}$ and $\mathrm{Ku}$ (1996), OSI is defined as:

$$
O S I=0.5 \cdot\left[1-\left(\frac{\left|\int_{0}^{T} W S S(s, t) \cdot d t\right|}{\int_{0}^{T}|W S S(s, t)| \cdot d t}\right)\right]
$$

where $s$ is the position on the vessel wall, and $T$ is the cardiac cycle. The OSI index identifies those regions on the vessel wall which show high oscillating WSS values during the cardiac cycle. In these regions bifurcating flows and vortex formation, which are related to atherosclerotic plaque formation and fibro-intimal hyperplasia (Giddens et al., 1993), are likely to be found. Many authors use this definition to describe the oscillation of vascular flows. Suchan oscillation varies from 0 to 0.5 . The areas where the WSS vector changes direction frequently show high OSI values. OSI index is not sensitive to the WSS magnitude but it is useful to identify regions with reversal flow. In this work, only WSS-based indices are taken into account. To compute these indices three components of the WSS vector are necessary. During a traditional diagnostic examination, it is quite impossible to obtain them from a specific patient. So, it is necessary to extract them by using different techniques and computational tools, like the CFD. 


\section{Practical insight: knowledge extraction for automation}

\subsection{D vs. $3 D$ simulation}

An initial survey was carried out considering the three-dimensional benchmark geometry and its corresponding two-dimensional mid-plane extraction surface. $\mathrm{Re}=250$ and $\mathrm{Re}=450$ are the two blood velocities taken into account for the numerical comparison. Subsequently, a systematic analysis was performed by varying both the geometry of the parametric CAD model, such as the diameter of the LCA, LAD, LCX sections, the diameter of the aneurysm, and flow characteristics. For each configuration tested, we monitored the average and maximum velocity that are established inside the blood vessel. We also sought a correlation between the variables monitored in 2D and 3D simulations. In particular, we have defined the following ratio:

$$
K=1-\frac{G_{2 D}}{G_{3 D}}
$$

where with $G_{2 D}$ we define the generic scalar monitored in a 2D simulation and $G_{3 D}$ is the same parameter monitored in a 3D simulation. Figure 4 shows how the 2D simulation underestimates the maximum velocity inside the vessel; by contrast $2 \mathrm{D}$ simulation overestimates the average velocity. The numerical results highlight that it is difficult to estimate a medium correlation constant as corrective factor useful to simplify the problem to bring the simulation from a 3D level to a $2 \mathrm{D}$ level. What it is highlighted is the strong dependence on the correlation constant from geometric parameters on the monitored scalars. For example, the diameter of the aneurysm has a considerable impact on the value of the constant based on the average velocity, while the average diameter of inlet (LCA) affects the value of the constant velocity.

Figure 4 Correlation constant $(\mathrm{K})$ for maximum and average velocity inside the vessel for different geometry configurations design process
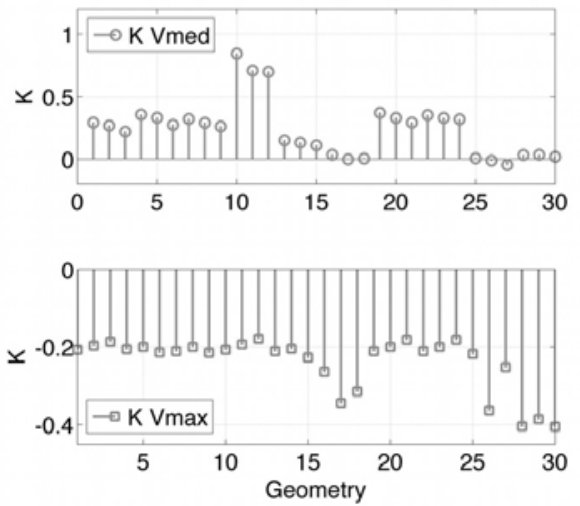


\subsection{Grid verification}

A 3D case is considered as a numerical benchmark on which numerical models are tested; unfortunately, given the geometric simplification compared to a real case, we do not have experimental data to make even the validation phase with the verification phase. As a result, it was made an intensive study of the verification phase of the grid. The grid verification is performed by using the verification procedure explained in Roache (1994), AIAA (1998). The reference value for the verification procedure is the base grid size used to perform the simulation. One finer $(\mathrm{GOH})$ and two coarser grids $(\mathrm{G} 1 \mathrm{H}, \mathrm{G} 2 \mathrm{H})$ were tested for non-conformal hexahedral discretization, Table 3, and two finer (G0P, G1P) and two coarse grids (G2P, G3P) were tested for polyhedral discretization, Table 4.

Table 3 Grid size for non-conformal hexahedral grids

\begin{tabular}{lc}
\hline Non-conformal hexa & $C V$ \\
\hline$G O H$ & 1.767 .844 \\
$G 1 H$ & 981.894 \\
$G 2 H$ & 371.920 \\
\hline
\end{tabular}

Table 4 Grid size for polyhedral grids

\begin{tabular}{lc}
\hline Polyhedral & $C V$ \\
\hline$G O P$ & 4.254 .911 \\
G1P & 1.602 .206 \\
G2P & 482.203 \\
G3P & 170.503 \\
\hline
\end{tabular}

Figure 5 shows the computed maximum velocity at $\mathrm{Re}=250$ with non-conformal hexahedral, divided by the values obtained with the grid $\mathrm{G} 0 \mathrm{H}$, versus the relative step sizes. The uncertainty bars showed in the figure are at $95 \%$ of confidence level. The uncertainties were derived using the guidelines in Stern et al. (2006).

Figure $5 \mathrm{Re}=250$, grids $\mathrm{G} 0 \mathrm{H}-\mathrm{G} 1 \mathrm{H}-\mathrm{G} 2 \mathrm{H}$; maximum velocity ratio vs. spatial resolution

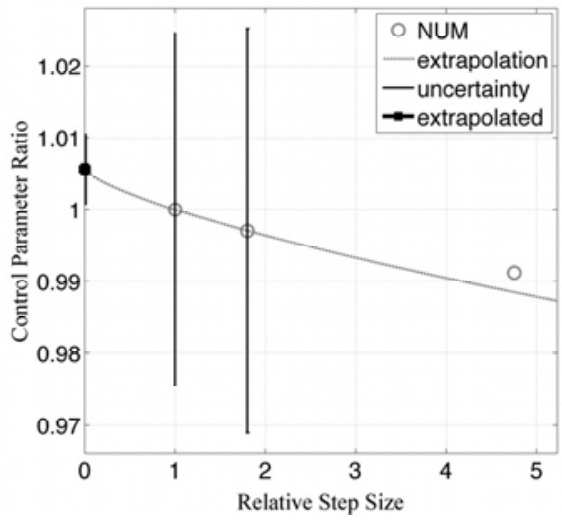


For the simulations with non-conformal hexahedral grids, we obtain an asymptotic convergence solution. Using a finer grid as $\mathrm{G} 0 \mathrm{H}$ the uncertainty prediction of the maximum velocity inside the vessel is $2.4 \%$ at $95 \%$ confidence, while with a medium grid $(\mathrm{G} 1 \mathrm{H})$, the uncertainty is $2.8 \%$. Extrapolating the results, an infinitely refined grid can perform a simulation with an uncertainty of $0.28 \%$ on the prediction of the maximum velocity. Computations carried out with polyhedral grids, using grids G1P, G2P and G3P, showed an oscillatory type convergence of the solution with the grid. To check the validity of oscillatory convergence it was tested further finer grid (G0P).

Figure 6 shows results of convergence with respect to the new fine grid: the convergence is not verified. The non-conformal hexahedral grids type tested at $\operatorname{Re}=250$, were tested at $\mathrm{Re}=450$ to check, with other values of blood velocity; the prediction of monitor sizes is in the asymptotic convergence range of the grid. Figure 7 shows the results of numerical/statistical survey; with grid $\mathrm{GOH}$, it is made an uncertainty prediction of the $1.3 \%$ at $95 \%$ confidence, while with a G1H grid, the uncertainty is $1.5 \%$. To reduce the uncertainty, a finer grid should be used.

Figure $6 \mathrm{Re}=250$, grids G0P-G1P-G2P; maximum velocity ratio vs. spatial resolution

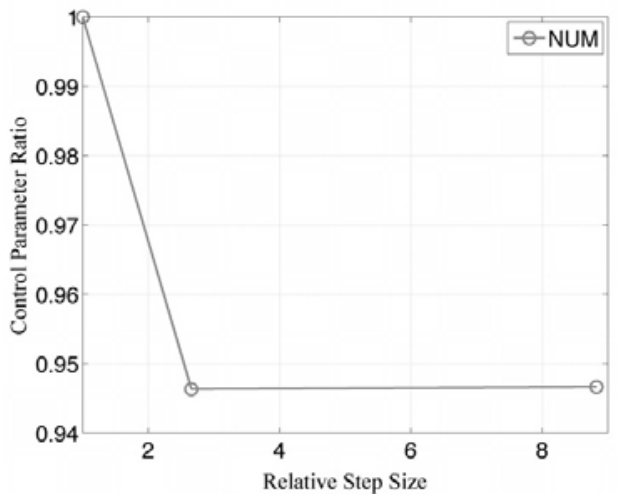

Figure $7 \mathrm{Re}=450$, grids $\mathrm{G} 0 \mathrm{H}-\mathrm{G} 1 \mathrm{H}-\mathrm{G} 2 \mathrm{H}$; maximum velocity ratio vs. spatial resolution

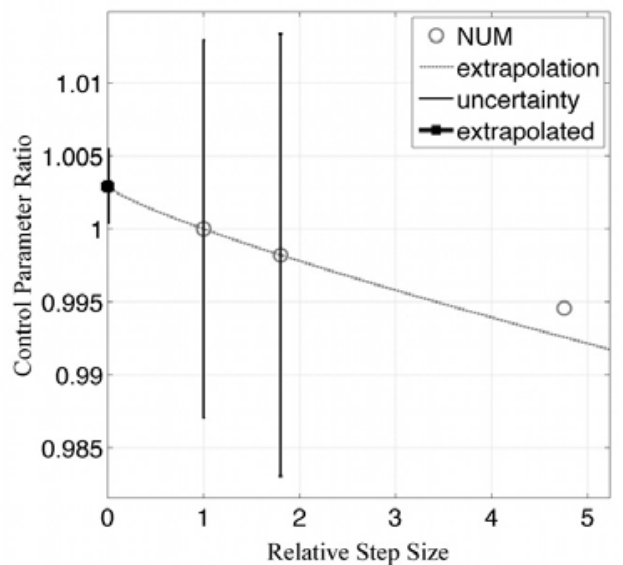


The analysis of the GCI indicates that the best choice to have an intelligent, robust and automatic pre-processing has to be based on non-conformal hexahedral grids with size of cells normalised by inlet diameter of 0.425 . Table 5 reports a resume of the extracted rules from the investigation.

Table 5 Rules extraction for the meshing knowledge

\begin{tabular}{lcc}
\hline Rule ID & Variable & Value \\
\hline$R 1$ & Grid type & Non-conformal hexahedral \\
$R 2$ & Base Cell size dx/D & Max 0.425 \\
$R 3$ & Surface size & Max $5 \%$ of dx/D \\
\hline
\end{tabular}

\subsection{Fluid viscosity}

In the discussion above, the CFD simulations were performed considering only blood as Newtonian fluid type. Additional simulations were performed considering fluid dynamic viscosity with the formulation of a non-Newtonian ones (De Gruttola et al., 2005). The physics characteristics of the fluid are shown in Table 6.

Table 6 Blood properties as non-Newtonian fluid

\begin{tabular}{lc}
\hline Density $\left[\mathrm{kg} / \mathrm{m}^{3}\right]$ & 1,050 \\
Power-law index, $n$ & 0.4851 \\
Consistency index, $k$ & 0.2073 \\
Maximum viscosity limit $[\mathrm{kg} / \mathrm{m}-\mathrm{s}]$ & 0.00125 \\
Maximum viscosity limit $[\mathrm{kg} / \mathrm{m}-\mathrm{s}]$ & 0.0035 \\
\hline
\end{tabular}

Figure 8 Comparison between Newtonian and non-Newtonian fluid numerical model at $\operatorname{Re}=250$ and $\operatorname{Re}=450$

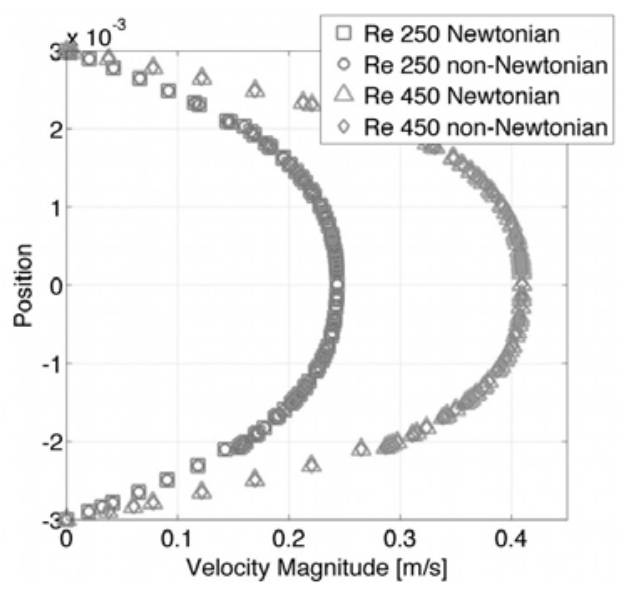

Two velocities up to $\mathrm{Re}=250$ and $\mathrm{Re}=450$ were tested with CFD simulations; the parameter monitored for the comparison of simulations with non-Newtonian fluid and Newtonian fluid is the velocity profile that occurs in aneurysm near the entrance. In both 
2D and 3D simulations it is clear that the dynamic viscosity of blood has much less influence than its influence on geometry characteristics, spatial discretization and domain dimension. Extracted knowledge from the investigation regards the fluid rheology model: the rule, for this specific case, (AAA) is to use a simple Newtonian model as a fluid viscosity model. Figure 8 shows a comparison between Newtonian and non-Newtonian fluid model.

\subsection{Influence of the boundary layer}

The generation of a mesh boundary layer which is able to detain the derived quantity object of interest is another key issue. The vast majority use fully unstructured isotropic meshes. The latter are not efficient from the point of view of computational time since a huge number of elements are required to solve the boundary layer at the wall properly and capture the derived quantities (i.e., WSS). Furthermore, these meshes may generate spurious fluctuations for the WSS (Sahni et al., 2006, 2009). On the contrary, boundary layer meshes are able to capture those quantities accurately and keep, at the same time, a reasonable number of elements for efficiency sake. It is possible to build such a mesh with an extrusion of the lumen surface in the inward direction. In this section, a systematic study of the influence of the boundary layer on the prediction of the WSS and velocity profile is presented. Simulations with different numbers of prism layers, from 4 to 12 , are compared with the simulation without the boundary layer. Mesh parameters are the same as the previous simulations and are equal for each one presented in this section. Three probe lines are used to extract the velocity profile for each simulation with different prism layer in the systolic and diastolic condition $(\operatorname{Re}=252$ and $\operatorname{Re}=450)$. Figures 9 to 10 present a sample of the velocity profiles from simulation with different boundary layers, for different blood flow conditions and different positions inside the vessel measured in a section downstream of the aneurysm in the LCX branch. It is evident how the boundary layer influences the prediction of the velocity profile near the wall of the vessel. Simulations without the boundary layer can produce a wrong prediction of the velocity profile, not only in the value of the quantity but also in the direction of the gradient of that, Figure 10. In presence of a bifurcation and particular features, like an aneurysm, the fluid flow can present a recirculation, in particular, when the flow changes direction according to the geometry. In these conditions, meshes without boundary layer anticipate the reattachment of the fluid flow inside the LCX branch. A wrong estimation of the flow field near the wall is also a wrong estimation of the WSS; as a result, a wrong estimation of those quantities can produce a risk index that is neither robust nor consistent. Figure 11 shows the comparison of the maximum WSS estimated by the simulations. For the two testing conditions, meshes without boundary layers underestimate the value of WSS. With only four prisms in the boundary layer, the prediction overestimates that quantity. A convergence in the prediction of the WSS is achieved with eight prisms in the boundary layer. More prisms in the boundary layer can make a more accurate prediction of the flow field near the wall, but increase the number of cells in the mesh and consequently, time effort and computational resource for a small increase of the quality in the prediction. Knowledge and the rule regarding the prism layer are extracted; in the prism layer near vessel walls, a minimum of eight prisms is necessary for the good resolution of the WSS. 
Figure 9 Normalised velocity profile for different prism layer in a section downstream the aneurysm in LCX branch

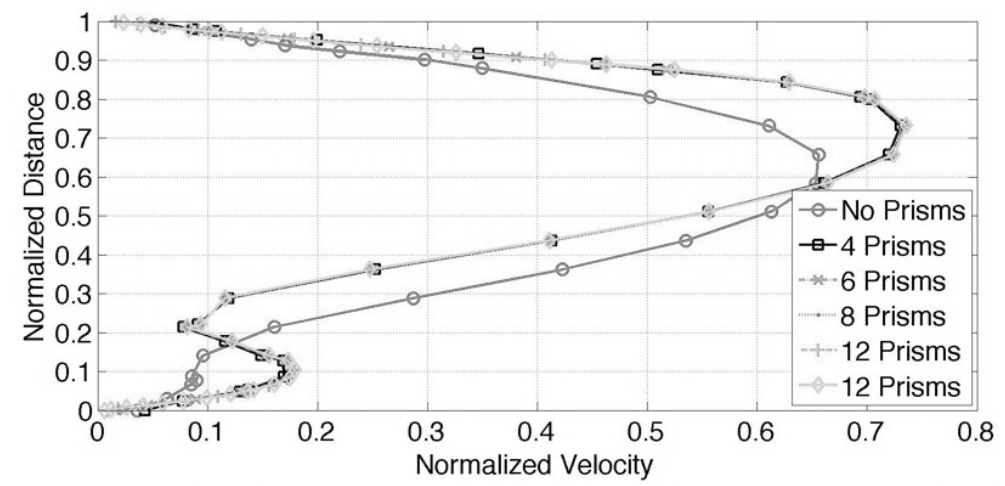

Figure 10 Particular of the normalised velocity profile near the wall: grids without boundary layer can lead to a wrong estimation of the velocity and also of the gradient

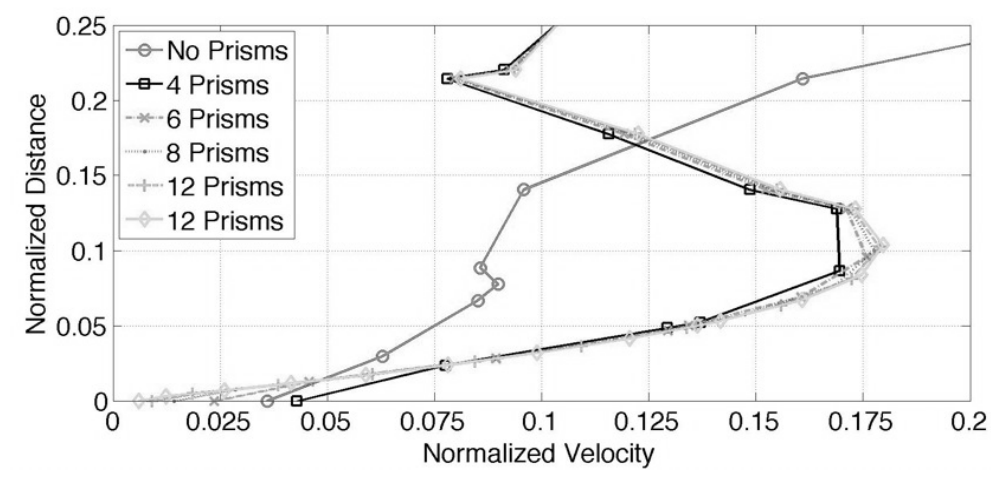

Figure 11 Maximum WSS magnitude inside the vessel with different numbers of prisms at Re wall

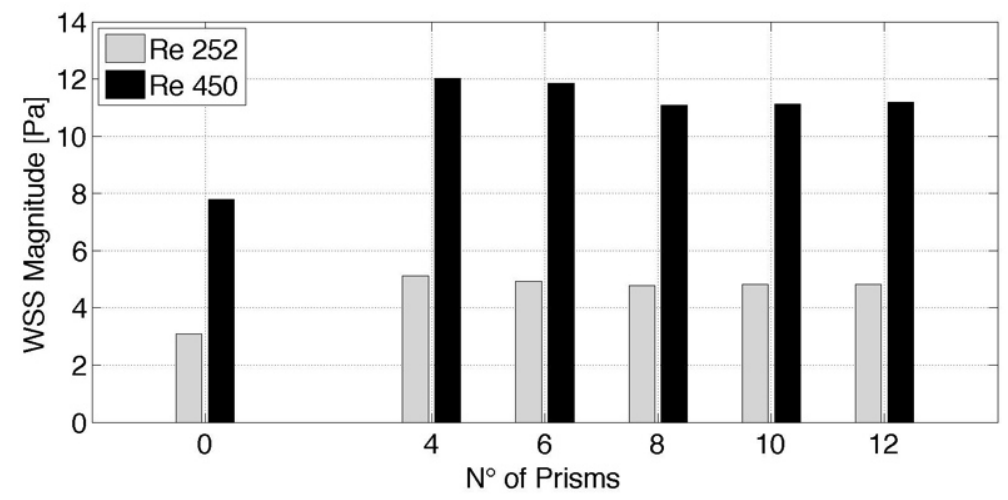




\subsection{Influence of the entrance length}

Considering a real case, the bifurcation is not truncated at both ends but is connected to the circulatory system. For this reason it was decided to study a new geometry from a reconstruction based on DICOM images. To have a similar condition to the real ones, a first amendment concerns the extrusion of the inlet and outlet regions, in order to give the model a developed velocity profile. Flow extensions are cylindrical extensions added to the inlets and outlets of a model. They are important for ensuring that the flow entering and leaving the computational domain is fully developed, so that fully developed boundary conditions do not force the solution in the actual vessel. Adding flow extensions is a typical problem in CFD modelling. Stitching cylindrical flow extension to an inlet or outlet of a realistic vessel is not a trivial task, and may result in worsening the reproducibility and adding operator-dependence to the modelling procedure. A fully automatic procedure can solve this - often overlooked - problem preserving reproducibility and speeding up the modelling phase considerably.

The first step in changing the geometry is to understand which is the entrance length that produces a fully developed flow in the axial direction. The reference geometry for this systematic study is a circular pipe of diameter $D=0.2 \mathrm{~m}$ and length $L=16 \mathrm{~m}$ subject to a Poiseuille flow at $\mathrm{Re}=100$. To extend the range of validity of the test case, other simulations were set with the same mesh and solver setup but with input conditions characterised by $\mathrm{Re}=252$ and $\mathrm{Re}=450$. For each of the two runs we extrapolated the value of the axial velocity along the pipe axis. The purpose is to check the entrance length of developed flow (Le) in which the maximum speed does not vary more according to the axial dimension, considering the flow as fully developed. This makes it possible to obtain the ratio of the extrusion length $\mathrm{L}$ to $\mathrm{Re}$, which guarantees that condition.

Figure 12 shows the development of the axial velocity inside the pipe for the three Re numbers considered. The value of Le is different for each of the Re. In Table 7, it is presented a resume of the results as well as the rules and knowledge for the extrusion length ratio between Le and Re.

Figure 12 Axial velocity development and position of the fully developed entrance length

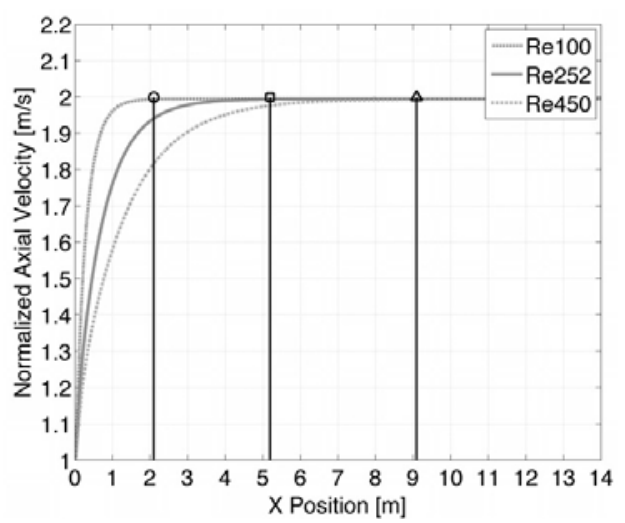


Table 7 Entrance length, Le, and extrusion length ratio, Le/Re

\begin{tabular}{lcc}
\hline & $L e[m]$ & $L e / R e$ \\
\hline $\operatorname{Re}=100$ & 2.10 & $21 \%$ \\
$\operatorname{Re}=250$ & 5.19 & $20 \%$ \\
$\operatorname{Re}=450$ & 9.09 & $20 \%$ \\
\hline
\end{tabular}

By using the rule extracted from the survey of the case test subject to Poiseuille flow, two simulations were developed on a real reconstructed geometry. Comparing the numerical simulations on the same geometry with and without developing boundary conditions, Figures 13 to 14, it is clear the velocity distribution is different within the aneurysm sac. Consequently, the distribution of WSS on the wall of the blood vessel also varies; in the case without developed flow, the maximum value relating to WSS is equal to WSSmax $=18.7 \mathrm{~Pa}$, while for the case with developed flow conditions it is equal to WSSmax $=18.9 \mathrm{~Pa}$.

Figure 13 Numerical results from the original geometry
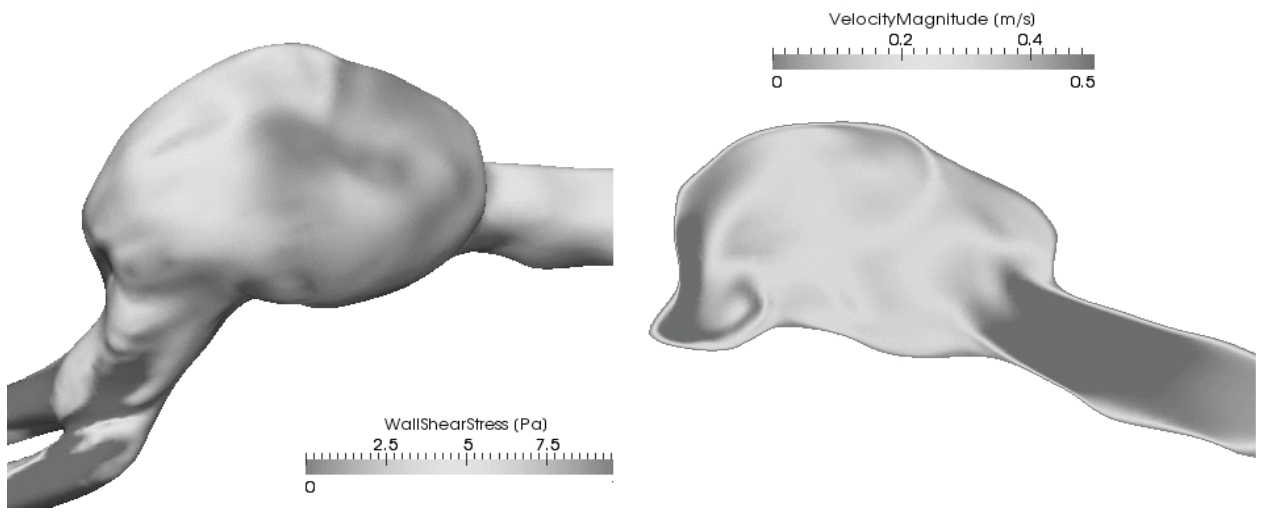

Figure 14 Numerical results from geometry with extrusion of the inlet and outlet boundary conditions
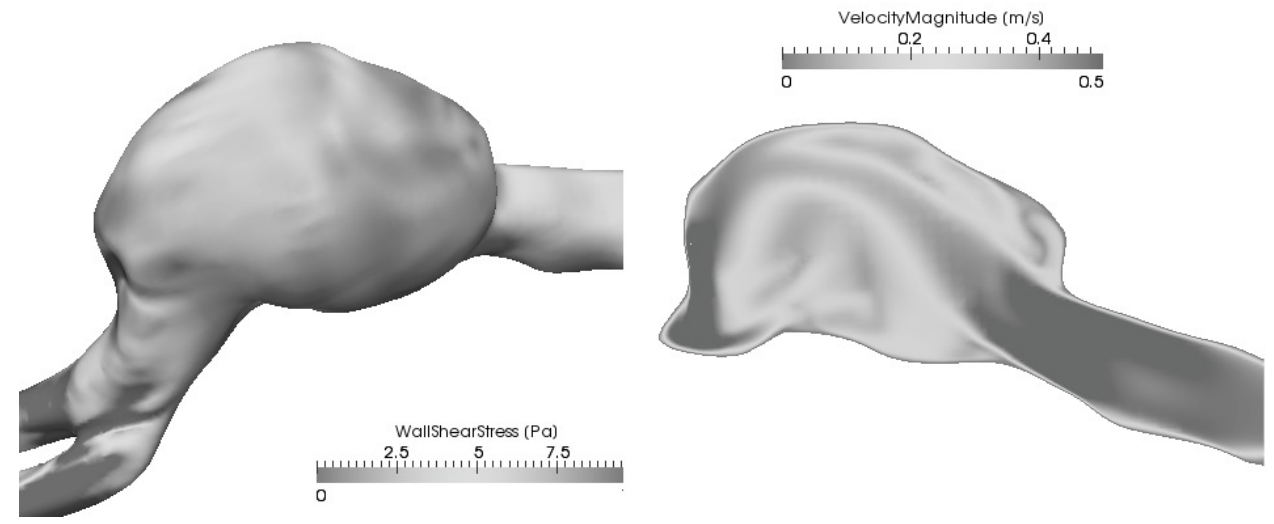
The comparison shows a difference between the two solutions in the prediction of WSSmax and WSSmax equal to $1 \%$. It is necessary to underline that the simulations were carried out with stationary speed input and flat profile. In the case of transitional regime, with input conditions that vary over time, as the cardiac cycle, the absence of fully developed flow amplifies the incorrect fluid dynamics within the aneurysmal sac, leading to an incorrect prediction of the value linked to the efforts on the wall.

\section{Clinical rules applications}

The rules extracted from the benchmark test case were tested by using geometries of real abdominal aorta bifurcation reconstructed from DICOM images. Two different geometry were reconstructed from two different clinical situation of two patients: the first one, called $P 1$ is a simple bifurcation with no evidence of risk of compliance; the second one, called $P 4$, is a bifurcation with the presence of a big aneurysm.

Appling the knowledge and rules extracted from the above investigation, the mesh generation can be done in compliance to the geometric properties of the two bifurcations. The main variable rule for the grid generation is the value of the reference length size of the single volume cell, based on aorta diameter, $x / D$. The value of the aorta diameter is extracted by using the geometric analysis performed during the anatomy reconstruction. So, it is possible to compute the mesh base size, for each patient analysed. This is the value at the base of the grid generation process. Setting up the rule inside the mesh generation, it is possible to generate a mesh suitable for AAA CFD simulations. As a result, for patient $P 1$, where the inlet aorta diameter is $21.4 \mathrm{~mm}$, the maximum cell size in the mesh is $9.1 \mathrm{~mm}$ and on vessel surface is $0.455 \mathrm{~mm}$; for patient $P 4$, where the inlet aorta diameter is $20.5 \mathrm{~mm}$, the maximum cell size in the mesh is $8.7 \mathrm{~mm}$ and on vessel surface is $0.435 \mathrm{~mm}$. Using the rules extracted above, the volumetric mesh size, with rules-based extruded boundary conditions and 8 prisms in the boundary layer, for the P1 case is $832.000 \mathrm{CV}$ and for $\mathrm{P} 4$ case is $812.000 \mathrm{CV}$.

Figure 15 Cardiac cycle measured from in-vivo experimentation

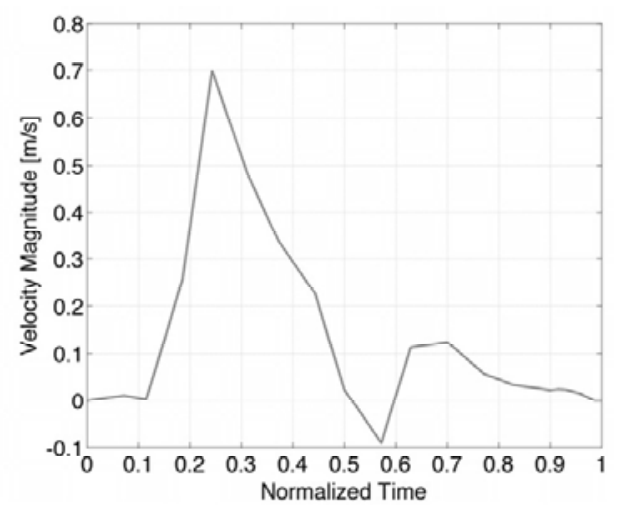

Source: Alamanni et al. (2012) 
Figure 16 OSI distribution and velocity magnitude inside the abdominal bifurcation of patient P1

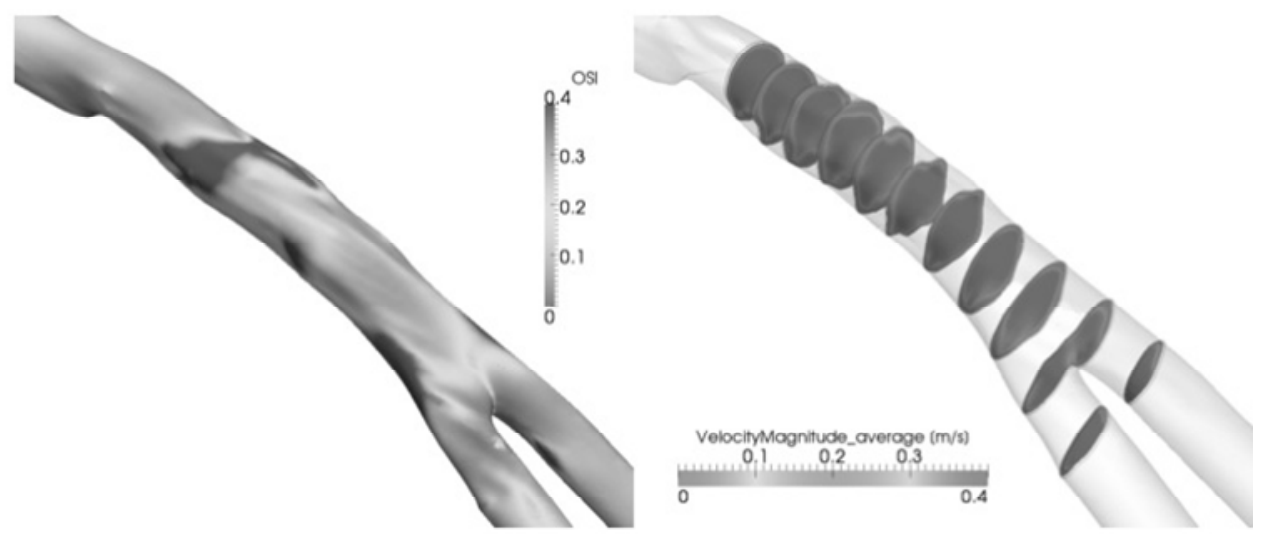

Figure 17 OSI distribution and velocity magnitude inside the abdominal bifurcation of patient P4
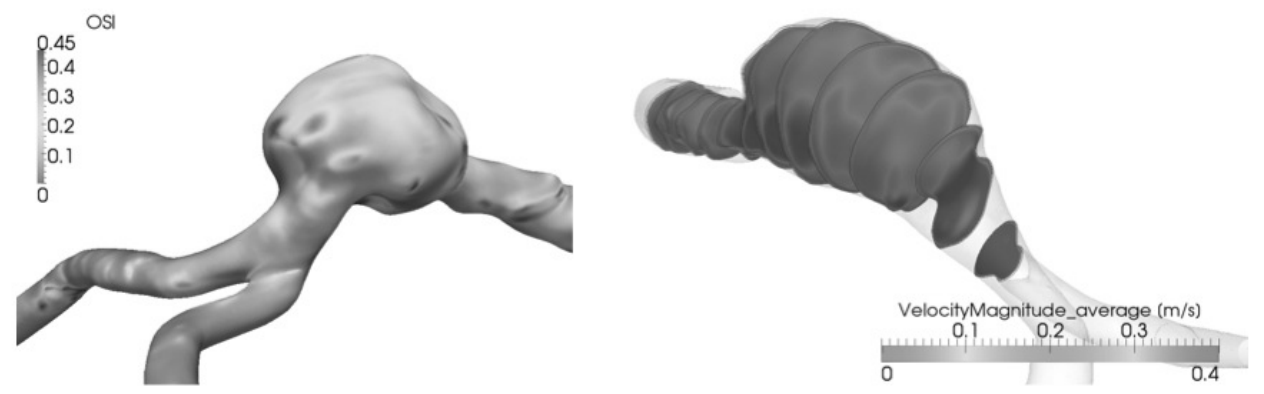

CFD simulations with unsteady formulation, Newtonian formulation for blood rheology and custom boundary conditions were implemented to analyse the fluid flow inside the vessels. Figure 15 shows the cardiac cycle used as boundary conditions for the solver comparison. According to the theory, by using the haemodynimics indices it is possible to have a visualisation of the compliance risk. Integrating in time the WSS components, it is possible to have a visualisation of the distribution of OSI and the average value of the velocity magnitude. Figures 16 to 17 show the hemodynamic index OSI distribution on the vessel walls of the bifurcation and the velocity distribution.

A preliminary comparison between two different CFD solvers was done by using the benchmark test case with the real cardiac cycle as boundary conditions. The investigation aims at extracting knowledge about the use of a specific solver for AAA cases. Commercial code Star-CCM+ and open-source libraries Open FOAM are considered; OpenFOAM is an open-source $\mathrm{C}++$ toolbox for the development of customised numerical solvers, including CFD.The setup for Star-CCM+ was the same as the previous simulation using an unsteady formulation with 1 st order of discretization in time. OpenFOAM was run using the pimpleFoam solver. The time history, Figure 18(a) to Figure 18(c) show differences in the flow field inside the vessel, but the average value, Figure 18(d), is comparable between the two solvers. Since an average value over the cardiac cycle is necessary for the haemodynamics indices calculation, open-source tools 
can be useful tools to use in biomedical fields because users can create custom objects and custom solvers to be embedded in an automatic work flow like the risk evaluation of an aneurysm.

Figure 18 Comparison between commercial solver Star-CCM+ and open-source solver OpenFOAM pimple Foam, $(\mathrm{a}, \mathrm{b}, \mathrm{c})$ are the instantaneous distribution of the velocity magnitude, according to the cardiac cycle (d) is the average distribution in time of the same scalar quantity

\section{Star-CCM+}

a

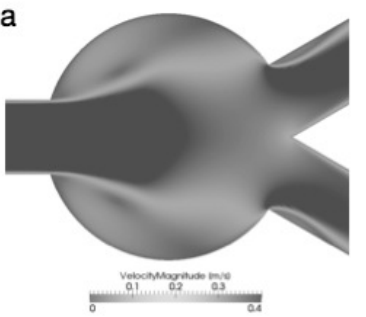

b

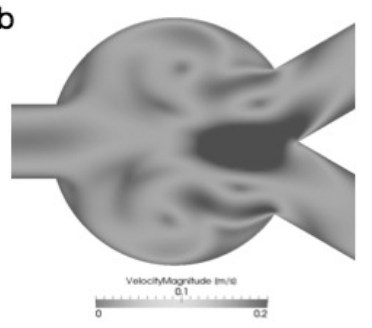

C

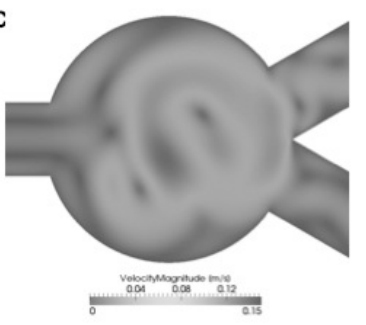

d

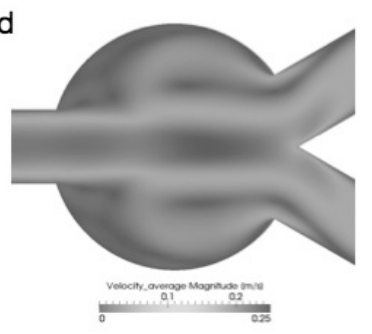

\section{OpenFOAM}
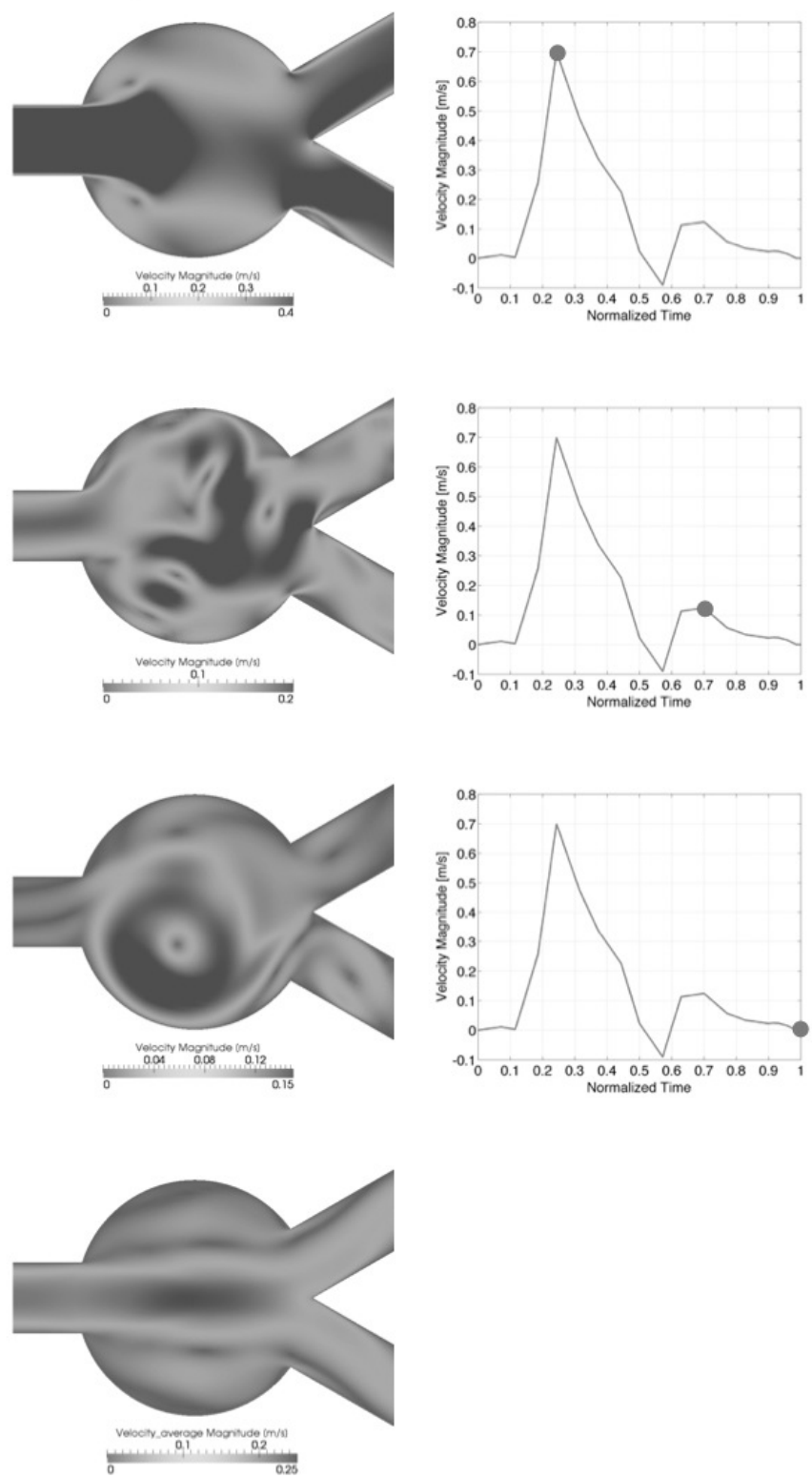


\section{Conclusions}

The work allows the assessment of geometries' effects, calculation grids, models and features of the fluid (blood) to determine some specific parameters (pressure, fluid velocity, tension and stress state of the vessel) in the case of an aorta aneurysm. Numerical results underlined the difficulty obtaining a constant correlation between 2D and 3D simulations. Besides, the numerical experimentation shows the tight interdependence between geometrical features and scalar quantities, such as the maximum velocity inside the vessel.

The investigation about grid generation brought to the definition of a discretization strategy, which is suitable to represent the physics closer to the real phenomenon. The use of structured grids allows the representation of the phenomenon with fewer numerical discretization errors than non-structural grids. As a consequence, the use of non-structural grids implies more grid refinement and then a bigger number of elements, which would require more time and resources. The grid convergence investigation, with $\mathrm{V} \& \mathrm{~V}$ procedure, showed that the grid refinement positively contributes to the prediction of scalar quantities, object of this study. Moreover, it allows obtaining a grid refinement threshold beyond which improvements - in terms of numerical results accuracy - are not possible. Concerning this geometry and these laminar flow conditions, the influence of the rheology model of the fluid is less than that of the geometric characteristics of the vessel as well as spatial discretization. It is necessary a good resolution at the boundary layer to compute the WSS correctly. A minimum of eight prisms was found as a good level of accuracy to compute well the fluid flow parameters. Moreover, the importance of the entrance length is not a minor issue in this field. The fluid flow needs to be fully developed before the entrance of the real vessel.

To sum up, the numerical investigation allowed obtaining the macro parameters to do an adequate CFD simulation on blood flows inside carotid bifurcations. A typical simulation for these types of problems is a 3D laminar simulation with Newtonian fluid and fine hexahedral grid. The results obtained allow designing an application that, in automatic mode and based on images taken from laboratory investigations, determines the abovementioned specific parameters, useful to choose the best therapy.

Future developments of this research will focus on further analysis of parameters of pre-processing and solving to make CFD simulations work in embedded mode. Besides, we will try to identify the most significant scalar quantities used in the medical field, for example to generate layouts with visual information useful for diagnosis. We will try to extract additional rules for the pre-processing focusing on the definition and imposition of boundary conditions, ensuring the opportunity to make any changes to the starter CAD geometry in relationship to the fluid flow inside the vessel. The solver part of the CFD process will be investigated to extract additional rules useful to do an automatic setup, such as the time step and the relative number of internal cycles for each time step. Finally, the research project will focus on the geometric reconstruction. Rules and procedure for this issue are to be obtained. The process will generate a CAD geometry from biomedical images, geometry will be the input data for the simulation process 'Embedded CFD.' 


\section{References}

AIAA (1998) Guide for the Verification and Validation of Computational Fluid Dynamics Simulations, Issue AIAA-G-077-1998, Am. Inst. Aeronaut, Astronaut, USA.

Alamanni, N., Lotti, M., Colombo, G., Chisci, E., Michelagnoli, S., Casetta C., Procacci, T., Pieri, A., Pugliese, D. and Bartesaghi, S. (2012) 'An automated procedure based on computer fluid dynamics to evaluate risk on abdominal aortic aneurysm', VPH 2012, London, UK, 18-20 September.

Andersson, P., Ludvigson, M. and Isaksson, O. (2006) 'Automated CFD blade design within a CAD system', Proceeding of the Integration of Numerical Simulation into the Product Development Process (FEA and CFD): 3rd NAFEMS Nordic Seminar, NAFEMS Contact Nordic Countries, Gothenburg, Sweden, pp.1-8.

Arghiropol, A. and Rotaru, C. (2010) 'Overview of the 2D and 3D finite element studies versus experimental results of a solid propellant engine performances under cycling loading effect', International Journal of Mathematics and Computers in Simulation, Vol. 4, No. 2, pp.42-49.

Arslan, N., Volkan Tuzcu, V., Nas, S. and Durukan, A. (2005) 'CFD modeling of blood flow inside human left coronary artery bifurcation with aneurysms', Proceeding of the 3rd European Medical and Biological Engineering Conference EMBEC'05, Prague, Czech Republic, Vol. 11, No. 1.

Botar, C.C., Vasile, T., Sfrangeu, S., Clichici, S., Agachi, P.S., Badea, R., Mircea, P. and Cristea, M.V. (2010) 'Validation of CFD simulation results in case of portal vein blood flow', in Pierucci, S. and Buzzi Ferraris, G. (Eds.): Proceeding of the 20th European Symposium on Computer Aided Process Engineering - ESCAPE20, Elsevier B.V., Ischia, Naples, Italy, pp.205-210.

Boutsianis, E., Guala, M., Olgac, U., Wildermuth, S., Hoyer, K., Ventikos, Y. and Poulikakos, D. (2009) 'CFD and PTV steady flow investigation in an anatomically accurate abdominal aortic aneurysm', Journal of Biomechanical Engineering, Vol. 131, No. 1, pp.011008.1-011008.15.

Celi, S., Di Puccio, F., Forte, P., Berti, S. and Mariani, M. (2009) 'Influenza dei Parametri Morfologici sul Rischio di Rottura dell'Aneurisma dell'Aorta', Proceeding of the AIAS-Associazione Italiana per l'Analisi delle Sollecitazioni, XXXVIII Convegno Nazionale, Torino, Vol. 400, pp.(149)1-(149)10.

Chapman, C.B. and Pinfold, M. (2001) 'The application of a knowledge based engineering approach to the rapid design and analysis of an automotive structure', Advances in Engineering Software, Vol. 32, No. 12, pp.903-912.

Cooper, C.A., Alderliesten, R., La Rocca, G. and Benedictus, R. (2009) 'In search of a knowledge based preliminary design method of complex aircraft wings', Proceeding of 7 th Annual Conference on Systems Engineering Research CSER 2009, Research School of Systems Engineering, Loughborough University, Loughborough, UK, pp.S10-63.

De Gruttola, S., Boomsma, K. and Poulikakos, D. (2005) 'Computational simulation of a non-Newtonian model of the blood separation process', Artificial Organs, Vol. 29, No. 12, pp.949-959.

Deschamps, T., Schwartz, P., Trebotich, D., Colella, P., Saloner, D. and Malladi, R. (2004) 'Vessel segmentation and blood flow simulation using level-sets and embedded boundary methods', International Congress Series 1268, USA, pp.75-80.

Ford, M.D., Nikolov, H.N., Milner, J.S., Lownie, S.P., DeMont, E.M., Kalata, W. and Loth, F. (2008) 'PIV-measured versus CFD-predicted flow dynamics in anatomically realistic cerebral aneurysm models', Journal of Biomechanical Engineering, Vol. 130, No. 2, pp.021015.1-021015.9.

Giddens, D.P., Zarins, C.K. and Glagov, S. (1993) 'The role of fluid mechanics in localisation and detection of atherosclerosis', ASME J. Biomech. Eng., Vol. 115, No. 4B, pp.588-594.

Grindberg, L., Cheever, E., Anor, T., Madsen, R. and Karniadakis, G.E. (2011) 'Modeling blood flow circulation in intracranial arterial networks: a comparative 3D/1D simulation study', Annals of Biomedical Engineering, Vol. 39, No. 1, pp.297-309. 
He, X. and Ku, D.N. (1996) 'Pulsatile flow in the human left coronary artery bifurcation: average conditions', Journal Biomech Engineering, Vol. 118, No. 1, pp.74-82.

Huber, A., Tang, W., Flowe, A., Bell, B., Kuehlert, K. and Schwaarz, W. (2004) 'Development and applications of CFD simulations in support of air quality studies involving buildings', Proceeding of 13th Joint Conference on the Applications of Air Pollution Meteorology with the Air \& Waste Management Association, Vancouver, British Columbia, Canada, J2.2.

Jung, J. and Hassanein, A. (2008) 'Three-phase CFD analytical modeling of blood flow', Medical Engineering \& Physics, Vol. 30, No. 1, pp.91-103.

Kakimpa, B., Hargreaves, D. and Owen, J.S. (2009) 'The flight of wind borne debris: an experimental, analytical, and numerical investigation - part III: CFD simulations', Proceeding of APCWE-VII the Seventh Asia-Pacific Conference on Wind Engineering, W1-C Windborne Debris, Taipei, Taiwan.

Karmonik, C., Klucznik, R. and Benndorf, G. (2008) 'Comparison of velocity patterns in an AComA aneurysm measured with 2D phase contrast MRI and simulated with CFD', Technology and Health Care, Vol. 16, No. 2, pp.119-128.

Longest, P.W. and Vinchurkar, S. (2006) 'Effects of mesh style and grid convergence on particle deposition in bifurcating airway models with comparisons to experimental data', Medical Engineering \& Physics, Vol. 29, No. 2, pp.350-366.

Malek, A.M., Alper, S.L. and Izumo, S. (1999) 'Hemodynamic shear stress and its role in atherosclerosis', J. Am. Med. Assoc., Vol. 282, No. 21, pp.2035-2042.

Milašinović, D., Ivanovic, M., Tengg-Kobligk, H., Böckler, D. and Filipovic, N. (2008) 'Software tools for generating CFD simulation models of blood flow from CT images, and for postprocessing', Journal of the Serbian Society for Computational Mechanics, Vol. 2, No. 2, pp.51-58.

Mohammed, J., May, J. and Alavi, A. (2008) 'Application of computer aided design (CAD) in knowledge based engineering', Proceedings of The 2008 IAJC-IJME International Conference, Nashville, Tennessee, paper 83.

Moroz, L., Govoruschenko, Y. and Pagur, P. (2005) 'Axial turbine stages design: 1D/2D/3D simulation, experiment, optimization', Proceeding of GT2005 ASME Turbo Expo 2005: Power for Land, Sea and Air, Reno-Tahoe, Nevada, USA, GT2005-68614.

Navier, C.L.M.H. (1823) 'Mémoire sur les lois du mouvement des fluids', Mem. Acad. R. Sci., Vol. 6, pp.389-416, Paris.

Nawijn, M., van Tooren, M.J.L., Berends, J.P.T.J. and Arendsen, P. (2006) 'Automated finite element analysis in a knowledge based engineering environment', Proceedings of the 44th AIAA Aerospace Sciences Meeting and Exhibit, Reno, Nevada, AIAA, Reston, Virginia, pp.9-12.

Pegemanyfar, N.V.D., Bank, R., Zedda, M., Pfitzner, M. and Savary, N. (2008) 'Design methodologies and CFD methods for the development of low emission combustion systems in aero-engines', Proceeding of 8th. World Congress on Computational Mechanics (WCCM8) and 5th European Congress on Computational Methods in Applied Sciences and Engineering (ECCOMAS 2008), Venice, Italy, a3589.

Petkova, S., Hossain, A., Naser, J. and Palombo, E. (2003) 'CFD modelling of blood flow in Portal vein hypertension with and without thrombosis', Proceeding of the Third International Conference on CFD in the Minerals and Process Industries CSIRO, Melbourne, Australia, pp.527-530.

Roache, P. (1994) 'Perspective: a method for uniform reporting of grid refinement studies', ASME J. Fluids Eng., Vol. 116, No. 3, pp.405-413.

Sahni, O., Jansen, K. and Shephard, M. (2009) 'Automated adaptive cardiovascular flow simulations', Engineering with Computers, Vol. 25, No. 1, pp.25-36.

Sahni, O., Mueller, J., Jansen, K., Shephard, M. and Taylor, C. (2006) 'Efficient an isotropic adaptive discretization of cardiovascular system', Comp. Meth. Appl. Mech. Eng., Vol. 195, pp.5634-5655. 
Spiegel, M., Redel, T., Zhang, Y.J., Struffert, T., Horneggera, J., Grossman, R.G., Doerfler, A. and Karmonik, C. (2011) 'Tetrahedral vs. polyhedral mesh size evaluation on flow velocity and wall shear stress for cerebral hemodynamic simulation', Computer Methods in Biomechanics and Biomedical Engineering, Vol. 14, No. 1, pp.9-22.

Stern, F., Wilson, R. and Shao, J. (2006) 'Quantitative V\&V of CFD simulations and certification of CFD codes', Intl. J. Numerical Methods in Fluids, Vol. 50, No. 11, pp.1335-1355.

Stokes, G.G. (1845) 'On the theories of the internal friction of fluids in motion, and of the equilibrium and motion of elastic solids', Trans. Camb. Phil. Soc., Vol. 8, pp.287-305.

Valencia, A., Ledermann, D., Rivera, R., Bravo, E. and Galvez, M. (2008) 'Blood flow dynamics and fluid-structure interaction in patient-specific bifurcating cerebral aneurysms', International Journal for Numerical Methods in Fluids, Vol. 58, No. 10, pp.1081-1100.

Yilmaz, F. and Gundogdu, M.Y. (2009) 'Analysis of conventional drag and lift models for multiphase CFD modeling of blood flow', Korea-Australia Rheology Journal, Vol. 21, No. 3, pp.161-173.

\section{Symbols}

$\begin{array}{ll}\text { LCA } & \text { Left coronary artery } \\ \text { LAD } & \text { Left anterior descending } \\ \text { LCX } & \text { Left circumflex artery } \\ \text { KBE } & \text { Knowledge-based engineering } \\ \text { CV } & \text { Control volume } \\ \vec{v} & \text { Velocity vector [m/s] } \\ \rho & \left.\text { Density [kg/m }{ }^{3}\right] \\ p & \text { Pressure [Pa] } \\ t & \text { Time [s] } \\ \vec{g} & \left.\text { Gravity acceleration [m/s }{ }^{2}\right] \\ \vec{S} & \text { Angular strain rate } \\ \vec{\tau} & \text { Stress tensor [Pa] } \\ \tau_{0} & \text { Yield stress threshold [pa] } \\ M & \text { Momentum [Nm] } \\ F_{i} & \text { Forces acting on the fluid [N] } \\ \mu & \text { Dynamic viscosity [Pa-s] } \\ \mu_{0} & \text { Yielding viscosity [Pa-s] } \\ \eta & \text { Non-Newtonian viscosity [Pa-s] } \\ \gamma & \text { Shear rate } \\ n & \text { Non-Newtonian fluid constant } \\ \text { Re } & \text { Reynolds number } \\ \text { WSS } & \text { Wall shear stress [Pa] } \\ & \end{array}$

